\title{
Development of Posture Alert Device
}

\author{
Anurag Jaiswal, Pranav Ahuja, Pawan Shukla, Pooja V. Deshmukh \\ Electronics and Tele-Communication Engineering Department, \\ Bharati Vidyapeeth (Deemed to be) University College Of Engineering, Pune
}

\begin{abstract}
Incorrect posture is a wide spread bane in today's world. It can cause back problems due to long term pain and can lead to depression. Wearable technology often provides a way to realize this aim by measuring the body posture of the wearer and giving feedback to him on correct or incorrect posture. Therefore, to correct one's posture, a correct sitting and standing position has to be established and trained gradually. The posture monitoring device alert the user just in case of poor posture by sending a message on the smart phone and powering up the vibration motor which is attachedon the hardware device. The device is low cost and works for people of every age group.
\end{abstract}

Keywords:- Bending, Posture Correction, Bluetooth, Wearable Device.

\section{INTRODUCTION}

Many people have experienced neck, shoulder, and back pains in their lives. Millions of patients are suffering from back pains all around the world ${ }^{[1]}$ This problem has become one of the most important factors that contribute to overall health. This problem is very common in office workers. This pain is annoying to daily life and sleeps quality. It has been found that the major reason for back pain is improper posture. Bad posture includes swaying back, pelvic tilt, slouching and bending over which ends up in spinal wear and tear. Not only this but also it leads to feelings of depression, and a better chance of back injury. Generally, if a person is suffering from severe back pain, they are advised to undergo surgery. But surgery as we know is a very complex and expensive route.

\section{A. Proper Posture}

Posture is the alignment of body parts in relationship to one another at any given moment ${ }^{[2]}$. Posture of a body is defined because the position and orientation of the body segments with relevance a hard and fast organization. Poor spinal posture relates to the relative position of the body whereby the top and upper trunk of the body is in a very forward flexed position. Neutral posture is taken into consideration when the highest and upper trunk of the body is at zero degrees to the rest of the spine.Posture can be quantitatively defined by angular differences between the inflection points of the spine and deviation about the sagittal plane. The approximate normal angle ranges are displayed in the Table- 1 .

\begin{tabular}{|c|c|}
\hline Spinal Section & Approximate Normal Range \\
\hline Cervical (lordosis) & From $19.4^{\circ} \mathrm{To} 21.4^{\circ}$ \\
\hline Thoracic (kyphosis) & From $45.1^{\circ} \mathrm{To} 49.0^{\circ}$ \\
\hline Lumbar (lordosis) & From $39.7^{\circ} \mathrm{To} 40.0^{\circ}$ \\
\hline Sagittal Plane Deviation (torsion) & Approximate to $0^{\circ}$ \\
\hline
\end{tabular}

Table 1:- Approximate normal angle differences in each spinal section.

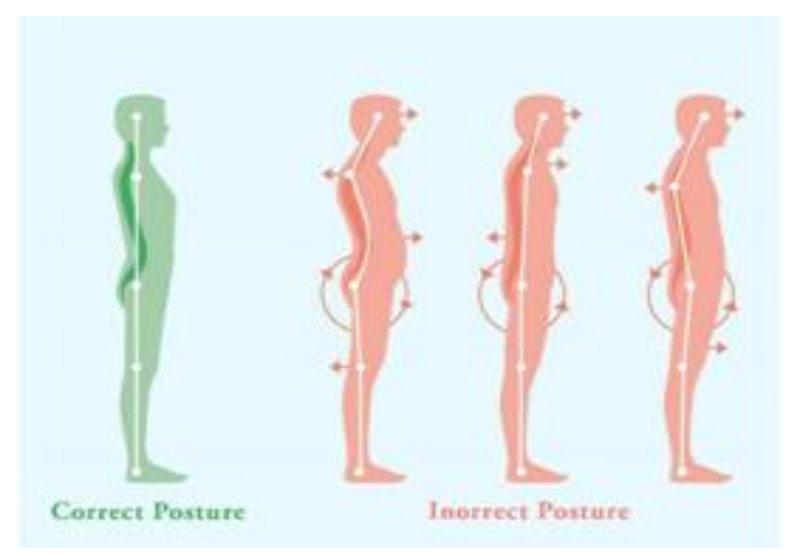

Fig 1: Correct and incorrect posture

\section{THEORY}

\section{A. Hardware}

1) $\mathrm{ESP} 32$

ESP32 is a series of cost-effective low-power systems on a chip microcontroller. It includes Wi-Fi and has a dualmode Bluetooth connectivity. The ESP32 series includes built-in components like antenna switches, power amplifiers, low-noise receiver amplifiers, filters, and power management modules. ${ }^{[3]}$ ESP32 is made and developed by Espressif Systems which is a Shanghai-based Chinese company, and it is manufactured by TSMC. TSMC used their $40 \mathrm{~nm}$ process. It was developed after the ESP8266 microcontroller.

ESP32 is meant for mobile, wearable electronics, and Internet-of-Things (IoT) applications. It features state-ofthe-art characteristics of low-power chips. It constitutes finegrained clock gating, multiple power modes, and dynamic power scaling. The low-duty cycle is employed to attenuate the quantity of energy that the chip expends. The output of the facility amplifier is additionally adjustable, thus contributing to an optimal trade-off between communication range, rate, and power consumption. 
ESP32 is a highly-integrated solution for Wi-Fi-andBluetooth based IoT applications. It has around 20 external components. As such, the whole solution occupies minimal computer circuit Board (PCB) area. It implements CMOS for single-chip fully-integrated radio and baseband connection, while also integrating advanced calibration circuitries which allows the output to induce obviate external circuit imperfections or unwanted changes in external conditions. ${ }^{[8]}$

\section{A.2 Accelerometer (MPU6050)}

The MPU6050 encloses a 3-Axis accelerometer and a 3-Axis gyroscope. All these components are integrated into one chip.

The gyroscope is used to measure rotational velocity or rate of change of the rotation in space over time, along the $\mathrm{X}$-axis, Y-axis, and Z-axis. Technologies like MEMS and Coriolis effect are used for measurement. The unit of a gyroscope output is in degrees per second, so as to urge the spatial relation angular velocity must be integrated. ${ }^{[4]}$

On the opposite hand, the MPU6050 accelerometer works same as an ADXL345 accelerometer sensor. Briefly, gravitational acceleration can be measured along the three axes, and sensor position angle can also be calculated using trigonometry maths. So, if we fuse, or combine the accelerometer and gyroscope data we'll get very accurate information about the sensor orientation. ${ }^{5]}$

The MPU6050 IMU is also called six-axis motion tracking device or 6 DoF (six Degrees of Freedom) devices, because of its 6 outputs, or the 3 accelerometer outputs and the 3 gyroscope outputs. ${ }^{[9]}$

\section{A.3 Coin Vibrating Monitor}

Coin vibration motoris also known asshaft less vibrator motor or pancake vibrator motor. These motors are generally in $8 \mathrm{~mm}-12 \mathrm{~mm}$ diameter. These motors are very compact and convenient to use. Vibration motors are widely utilized during a spread of applications including cell phones, handsets, pagers, and so on. ${ }^{[6]}$

Enclosures can easily be molded to accept the coin style of theshaft less vibration motors. Within the coin motor range, lead springs and pad mounted versions are provided.

The coin or pancake vibrating motors are Eccentric Rotating Mass (ERM) motors. Therefore they are be driven within the same manner as their pager motor counterparts. they need constant motor drive principles, including $\mathrm{H}-$ bridge circuitry for active braking.

The vibration motor rotor consists of two voice coils and a bit mass. This all is integrated into a flat plastic disc and has an impression within the center, which sits on a shaft. Two brushes on the underside of the plastic disc make contact with the PCB commutation pads and this supplies power to the voice coils and a force field is generated. This field interacts with the flux generated by a disc magnet that's attached to the motor chassis.
The commutation circuit alternates the direction of the sector through the voice coils, and this interacts with the N-S pole pairs that are built into the neodymium magnet. The disc rotates and, because of the built-in off-centred eccentric mass, the motor vibrates. ${ }^{[10]}$

\section{B. Software}

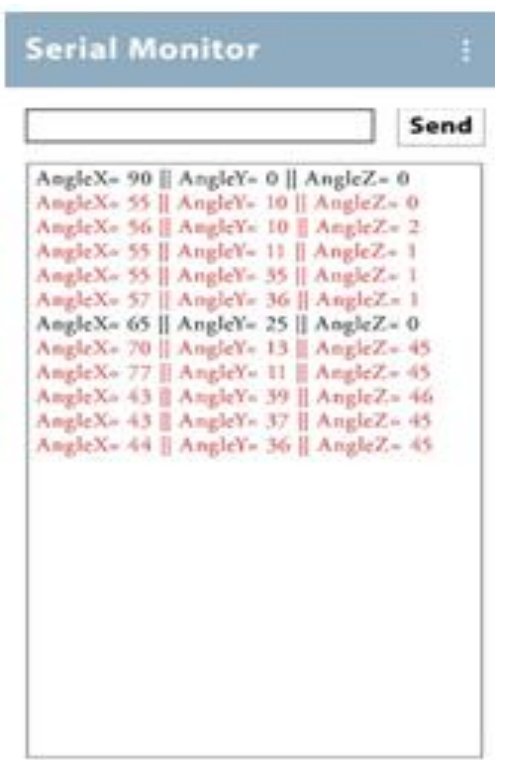

Fig.2:- Serial monitor on Mobile Phone

In this project we are using esp32 to transfer data to the mobile phone using Bluetooth. The data measured using the esp32 is sent to the mobile phone. The mobile phone has an application which reads the data and display the values. These values are judged and the values which led the device to vibrates are automatically displayed in red color. This application also keeps record of how many times a person was in bad posture.

The Fig.2. shows the working of that application. From the figure we know that the angle of $\mathrm{X}$ axis is should be between $60^{\circ}$ and $120^{\circ}$, the angle of $\mathrm{Y}$ axis should be between $330^{\circ}$ and $30^{\circ}$ and the angle of $\mathrm{Z}$ axis should be between $330^{\circ}$ and $30^{\circ}$.

\section{Block Diagram}
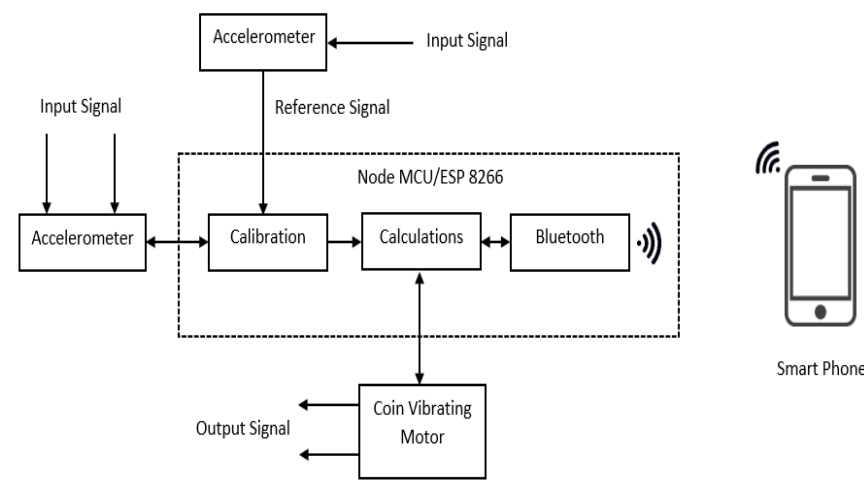

Smart Phone

Fig.3 Block-diagram of the proposed methodology 
The blocks of the block diagram in the proposed methodology(Fig.3) has been explain as follows:-

1. Accelerometer which is responsible for monitoring the orientation of the device and also the human body. Its uses piezo-electric effect to monitor the orientation. It has three axis for orientation. It measures the angle of inclination in all three axis; X-Axis, Y-Axis, Z-Axis.

2. Calibration gives the default value to which the accelerometer should be calibrated. This value is stored in the memory and is responsible for resetting the accelerometer value.

3. Calculations gives the information about parameter calculation such as angle of inclination and the degree of bending. It measures the appropriate angle to which the inclination can be increased.

4. WI-FI Module is the structure for communication with the mobile phones over a wireless network. It generated a hotspot signal and is connected by the mobile phone.

5. Vibration Motor is a module for indicating the user about its wrong position while any sort of work. It is responsible for receiving signal from the calculation block and produce a vibration if the angle is more that legitimate angle allowed.

6. Smart Phone is the device which is also used as a display screen for displaying the value of inclination angle. It is connected to the Node MCU wirelessly.

\section{Working}

There is a case containing all of these items in it. This case is placed on the top area of the spinal cord. This case has all the elements in it and also contains a charging port which is used to charge the battery. We will use the lithium ion battery. One accelerometer will measure the orientation of the body and other will set the default value for the previous accelerometer. They will do this by using piezoelectric effect.

This value is then sent to the calibrator which decides whether this value is in the calibrator range or not. If not then it sends the signal to the calculation block. This block will calculate the angle of inclination based on different parameters. After this the signal is sent to the wifi module of the Node MCU.

This module passes this signal to the smart phone connected to it to display the values of that angle. If that certain angle is maintained for more than a specific period of time then the smart phone will send the signal to the vibration sensor via wifi module and calculation block. This vibration module will produce vibration signal that will inform the user to correct the posture position.

\section{CONCLUSION}

In this paper, we propose a system to help the user in correcting their posture by real-time feedback, which is done through our personalized mobile application. In terms of working, our device was able to work as expected. Through android application we can receive a feedback on the posture, so that one can monitor his/her sitting posture. By giving such a feature, users will be able to correct posture as we would define the correct sitting posture in the app itself. This is how when a we reach in a bad posture, the vibration motor and our app would indicate the change in the values and that in the posture too correctly. This device is very simple to use and is wearable also it is low cost.

\section{ACKNOWLEDGEMENT}

This work is supported by Department of Electronics and Communication Engineering, BVPCOE, Pune.

\section{REFERENCES}

[1]. ErissaIrani, RohitJha, and Amanda Pustis, "Cornell Posturecorrector,"Cornell University project.

[2]. https://www.sciencedirect.com/topics/biochemistrygenetics-and-molecular-biology/body-position

[3]. https://en.wikipedia.org/wiki/ESP32

[4]. https://en.wikipedia.org/wiki/Accelerometer

[5]. https://howtomechatronics.com/tutorials/arduino/ardui no-and-mpu6050-accelerometer-and-gyroscopetutorial/

[6]. Yangyi Chen, "Vibration Motor" issue 4, April 2013.

[7]. https://en.wikipedia.org/wiki/Arduino_IDE

[8]. Espressif System, "Esp32 series datasheet", October 8, 2016

[9]. InvenSense, "MPU 6050 datasheet", August 19, 2013

[10]. Jinlong Machinery, "Vibration Motor", May 18,2009 